\title{
Application of Soft Computing Techniques for Analysis of Vapour Compression Refrigeration System
}

\author{
D.V.Raghunatha Reddy ${ }^{\dot{A} *}$ P.Bhramara ${ }^{\dot{B}}$ and K.Govindarajulu ${ }^{\dot{C}}$ \\ ${ }^{\dot{A}}$ ICFAI Foundation for Higher Education, Faculty of Science and Technology, Hyderabad, India \\ $\dot{B}$ JNTUH College of Engineering Hyderabad, India \\ ${ }^{\mathrm{C}}$ JNTUA College of Engineering Ananthapuram, India
}

Accepted 10 January 2014, Available online 01 February 2014, Special Issue-2, (February 2014)

\begin{abstract}
In recent years, soft computing (SC) techniques have been widely used in the analysis of vapour compression refrigeration system (VCR). Soft computing is becoming useful as an alternate approach to conventional techniques. Soft computing differs from conventional (hard) computing in that; it is tolerant of imprecision, uncertainty, Partial truth and approximation. The techniques are evolutionary computing such as Artificial Neural Networks (ANN) and Fuzzy Logic $(F L)$ each technique can be used separately, but a powerful advantage of soft computing is the complementary nature of the techniques. Used together they can produce solutions to problems that are too complex can be solved with conventional mathematical methods. The applications of soft computing have proved two main advantages. First one it solving nonlinear problems, in which mathematical models are not available and second, it introduced the human knowledge such as cognition, recognition, understanding, learning, and others into the fields of computing. These results can give the intelligent systems such as autonomous self-tuning systems, and automated designed systems. However the computer simulation method has its advantages over the conventional one. With the computer simulation method, the working conditions and the configuration parameters of the product are given at first, then the performances predicted, and at last the configuration parameters of the product is evaluated based on the performance prediction. If the predicted performance does not meet the requirement, the configuration parameters can be adjusted, and Simulation will be done again. Hence soft computing has been widely used for performance prediction and analysis of vapour compression refrigeration system.
\end{abstract}

Key words: Soft Computing, Fuzzy Logic, Artificial Neural Network, vapour compression refrigeration system

\section{Introduction}

Soft computing is a wide ranging term encompassing such varied techniques as fuzzy systems and neural networks, simulated annealing etc. In this paper we are using only Fuzzy Logic (FL) and Artificial neural network (ANN) Techniques. FL and ANNs have been successfully used for supply chain modeling (2008) and are particularly appropriate for this problem due to their capacity to tackle the inherent vagueness, uncertainty and incompleteness of the data used. FLis based on fuzzy set theory and provides methods for modeling and reasoning under uncertainty, a characteristic present in many problems, which makes FL a valuable approach. It allows data to be represented in intuitive linguistic categories instead of using precise (crisp) numbers which might not be known, necessary Orin general may be too restrictive. These categories are represented by means of a membership function which defines the degree to which a crisp number belongs to the category. Soft Computing Techniques (Artificial Neural Networks, Genetic Algorithms, Fuzzy Logic Models, and

*Corresponding author: D.V.Raghunatha Reddy

DOI: http://dx.doi.org/10.14741/ijcet/spl.2.2014.67
Expert System) have been recognized as attractive alternatives to the standard, well established "hard computing" paradigms. Traditional hard computing methods are often too cumbering some for today's problems. They always require a precisely stated analytical model and often a lot of computational time. Soft computing techniques, which emphasize gains in understanding system behavior in exchange for unnecessary precision, have proved to be important practical tools for many contemporary problems. These are universal approximates of any multivariate function because they can be used for modeling highly nonlinear, unknown, or partially known complex systems, plants, or processes. The course contents will be taught by eminent experts in the field, having adequate teaching and research experience. This course will be beneficial to faculty from all engineering disciplines as a potential computing tool in their research activities.

\section{Soft Computing}

Soft Computing became a formal Computer Science area of study in early 1990s. Earlier computational approaches 
could model and precisely analyze only relatively simple systems. More complex systems arising in biology, medicine, the humanities, management sciences, thermal systems such as refrigerator, heat pump and airconditioning and similar fields often remained intractable to conventional mathematical and analytical methods. That said, it should be pointed out that simplicity and complexity of systems are relative, and many conventional mathematical models have been both challenging and very productive. Soft computing deals with imprecision, uncertainty, partial truth, and approximation to achieve practicability, robustness and low solution cost. Components of soft computing include: Generally speaking, soft computing techniques resemble biological processes more closely than traditional techniques, which are largely based on formal logical systems, such as sentential logic and predicate logic, or rely heavily on computer aided numerical analysis (as in finite element analysis). Soft computing techniques are intended to complement each other.

The two major problem-solving technologies include:

- Hard computing

- $\quad$ Soft computing

Hard Computing deals with precise models where accurate solutions are achieved quickly. On the other hand, soft computing deals with approximate models and gives solution to complex problems. Hard computing schemes, which strive for exactness and full truth, soft computing techniques exploit the given tolerance of imprecision, partial truth, and uncertainty for a particular problem. Another common contrast comes from the observation that inductive reasoning plays a larger role in soft computing than in hard computing.

Soft computing addresses problem solving tasks in a complementary approach more than in a competitive one. Main advantages of soft computing are: i) its rich knowledge representation (both at signal and pattern level), ii) its flexible knowledge acquisition process (including machine learning and learning from human experts) and iii) its flexible knowledge processing. These advantages let us to build intelligent systems with a high machine intelligence quotient at low cost.

In recent years a growing field of research in "Adaptive Systems" has resulted in a variety of adaptive automations whose characteristics in limited ways resemble certain characteristics of living systems and biological adaptive processes. An adaptive automation is a system whose structure is alterable or adjustable in such a way that its behavior and performance improves by its environment. A simple example of an adaptive system is the automatic gain control use $\mathrm{d}$ in radio and television receiver. The most important factor in adaptive system is its time-varying and self-adjusting performance. Their characteristic depends upon the input signal. If a signal is applied to the input of adaptive system to test its response characteristic, the system adapts to this specific input and there by changes its parameters. Based on the different neural architecture of human brain, different Artificial Neural Algorithms are developed such as Artificial Neural Network (ANN), Multi-Layer Perception (MLP), Radial Basis Function (RBF), Adaptive NeuroFuzzy Inference
System (ANFIS) etc. These are capable of mapping the input and output nonlinearly.

\section{Methodologies Used}

\subsection{Artificial Neural Network}

ANN (Artificial Neural Network) is an abstract simulation of a real nervous system that contains a collection of neuron units communicating with each other via axon connections. Recently, ANN has been found to be an important technique for classification and optimization problem. McCullochandPitts have developed the neural networks for different computing machines. There are extensive applications of various types of ANN in the field of communication and instrumentation control. The ANN is capable of performing nonlinear mapping between the input and output space due to its large parallel interconnection between different layers and the nonlinear processing characteristics. An artificial neuron basically consists of a computing element that performs the weighted sum of the input signal and the connecting weight. The sum is added with the bias or threshold and the resultant signal is then passed through a nonlinear function of sigmoid or hyperbolic tangent type. Each neuron is associated with three parameters whose learning can be adjusted the connecting weights, the bias and the slope of the nonlinear function. For the structural point of view a NN maybe single layer or it may be multilayer. In multi layer structure, there is one or many artificial neurons in each layer and for a practical case there may be a number of layers. Each neuron of the one layer is connected to each and every neuron of the next layer. The functional-link ANN is another type of single layer NN. In this type of network the input data is allowed to pass through a functional expansion block where the input data are nonlinearly mapped to more number of points. This is achieved by using trigonometric functions, tens or products or power terms of the input. The output of the functional expansion is then passed through a single neuron.

The learning of the NN may be supervised in the presence of the desired signal or it may be unsupervised when the desired signal is not accessible. Rumelhart developed the Back propagation (BP) algorithm, which is central to much work on supervised learning in MLP.A feed forward structure with input, output, hidden layers and nonlinear sigmoid functions are used in this type of network. In recent years many different types of learning algorithm using the incremental back propagation algorithm, evolutionary learning using the nearest neighbor MLP and a fast learning algorithm based on the layer by layer optimization procedure are suggested in literature. In case of unsupervised learning the input vectors are classified into different clusters such that elements of a cluster are similar to each other in some sense. The method is called competitive learning, because during learning sets of hidden units complete with each other to become active and perform the weight change. The winning unit increases its weights on those links with high input values and decreases them on those with low 
input values. This process allows the winning unit to be selective to some input values. Different types of NNs and their learning algorithms are discussed in sequel.

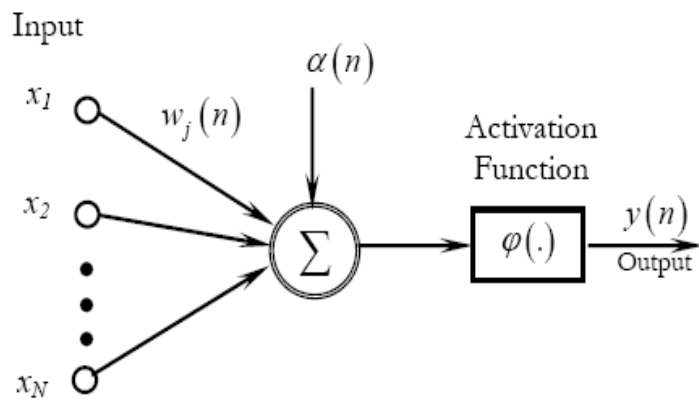

Fig 3.1: Structure of Neuron

The basic structure of an artificial neuron is presented in Fig. The operation in a neuron involves the computation of the weighted sum of inputs and threshold. The resultant signal is then passed through a nonlinear activation function. This is also called as a perception, which is built around a nonlinear neuron; whereas the LMS algorithm described in the preceding sections is built around a linear neuron. The output of the neuron may be represented as

$y(n)=\varphi\left[\sum_{j=1}^{N} w_{j}(n) x_{j}(n)+\alpha(n)\right]$

Where is the threshold to the neurons at the first layer, wj (n) is the weight associated with jth the input , $N$ is the number of inputs to the neuron and $\varphi($.$) is the nonlinear$ activation function.

\subsection{Fuzzy Logic}

Fuzzy logic is a valuable tool, which can be used to solve highly complex problems where Mathematical model is too difficult or impossible to create. It is also used to reduce the complexity of existing solutions as well as increase the accessibility of control theory. The development of software has always been characterized by parameters that possess certain level of fuzziness. Study showed that fuzzy logic model has a place in software effort estimation. The application of fuzzy logic is able to overcome some of the problems which are inherent in existing effort estimation techniques. Fuzzy logic is not only useful for effort prediction, but that it is essential in order to improve the quality of current estimating models. Fuzzy logic enables linguistic representation of the input and output of a model to tolerate imprecision. It is particularly suitable for effort estimation as many software attributes are measured on nominal or ordinal scale type which is a particular case of linguistic values. A method is proposed as a Fuzzy Neural Network (FNN) approach for embedding artificial neural network into fuzzy inference processes in order to derive the software effort estimates. Artificial neural network is utilized to determine the significant fuzzy rules in fuzzy inference processes. The results showed that applying FNN for software effort estimates resulted in slightly smaller mean magnitude of relative error (MMRE) and probability of a project having a relative error of less than or equal to 0.25 (Pred (0.25)) as compared with the results obtained by just using artificial neural network and the original model.

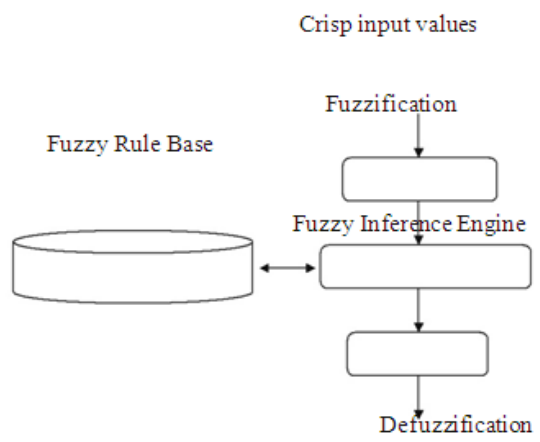

Crisp output values

Fig 3.2: Simple fuzzy system structure

A simple fuzzy system consists of four blocks: A Fuzzifier, Defuzzifier, inference engine and fuzzy rule knowledge base. Fuzzy Logic Controller (FLC) is an attractive choice when precise mathematical formulations are not possible. Other advantages are:

- It can work with less precise inputs.

- It doesn't need fast processors.

- It is more robust than other non-linear controllers

In this paper, we proposed a new method based on soft computing approach for FDD in Vapor Compression Refrigeration system. The schematic of the proposed method is depicted in Figure 1. As the figure suggests, the suitable feature vectors that are as inputs of TakagiSugeno (T-S) fuzzy classifier are calculated by applying the wavelet transform to the output signals and Reduction of redundant wavelet coefficients. Finally, the T-S fuzzy classifier that is tuned off-line by Differential Evolution algorithm detects and diagnoses the faults (if exist). The simulation has been done in MATLAB-Simulink.

\subsection{Principal of Vapour Compression Refrigeration}

Vapour Compression Refrigeration Systems (VCRS) that are used for different zones can be divided into four categories, namely, All-air systems, Air-water systems, All-water systems and Unitary or refrigerant-based systems. In the last category, vapor compression cycles are mostly used. Many of vapor compression cycles have been consisted of four components that are as follow.

Compressor: Commonly referred as the heart of system. It is responsible for compressing and transferring refrigerant gas.

Condenser: Is the area in which heat dissipation occurs. The condenser is designed to radiate the heat. As hot compressed gas is entered into the top of the condenser, it is cooled off. As the gas cools in condenses, it exits from the bottom of condenser as a high pressure liquid.

Evaporator: the evaporator serves as the heat absorption component. Its primary duty is to remove the heat from the zone. A secondary benefit is dehumidification. Refrigerant enters the bottom of the evaporator as a low pressure 
liquid. The warm air passing through the evaporator fins causes the refrigerant to boil. As the refrigerant begins to boil, it can absorb large amount of heat.

Throttling device: Is a device that allows a controlled amount of liquid refrigerant to enter into the evaporator from the condenser.

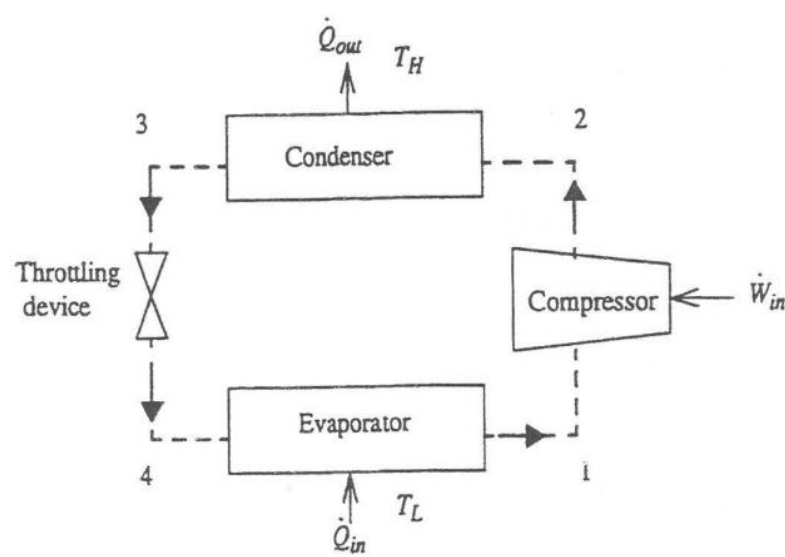

Fig3.3: Vapour Compression Refrigeration System

\section{Experimental Methodology}

This section provides a description of the facilities developed for conducting experimental work on a domestic refrigerator. The technique of charging and evacuation of the system is also discussed here. Experimental data collection was carried out in the research laboratory of our institution. The experimental setup of the test unit is given below

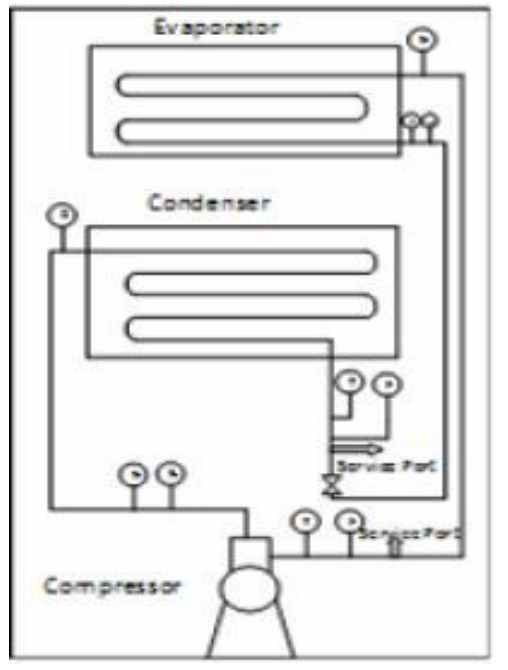

Fig4.1: Schematic diagram of the investigation unit

\subsection{Experimental Setup}

The experimental setup of the household refrigerator used in the experiment is shown in Fig 2. The domestic refrigerator consists of an evaporator, wire mesh aircooled condenser and hermetically sealed reciprocating compressor. The 185 liters domestic refrigerator of tropical class originally designed to work with HFC134awas taken for this study. The refrigerator was instrumented with one pressure gauge at the inlet of the compressor for measuring the suction pressure, one temperature sensor mounted at inside the refrigerator (freezer) compartment. As per the refrigerator manufactures recommendation quantity of charge requirement forHFC134a was $100 \mathrm{~g}$. In the experiment, refrigerant charge is $10 \%$ higher due to the presence of instruments and connecting lines etc. To optimize the mixed refrigerant charge, the refrigerator is charged with $80 \mathrm{~g}$. The refrigerator was charged with $110 \mathrm{~g}$ of HFC134a and the base line performance was studied. After completing the base line test with HFC134a, the refrigerant was recovered from the system and charged with $60 \mathrm{~g}$ of mixed refrigerant and the performance was studied. The refrigerant charge requirement with hydrocarbons is very small due to their higher latent heat of vaporization. During the experimentation the atmospheric is maintained at $26 \pm 2 \mathrm{oC}$. The experimental procedures were repeated and take the reading from the various modes of different loading conditions. Specially, we conduct the investigation is purely based on the vegetables in 0.5 and $1 \mathrm{~kg}$ load factor. Service port is installed at the inlet of expansion valve and compressor for charging and recovering the refrigerant is shown in Figure. Digital Temperature Indicator was used to measure the inside freezer temperature for this research.

\subsection{Test Procedure}

The system was evacuated with the help of vacuum pump to remove the moisture and charged with the help of charging system. The temperature inside the chamber was maintained at $25^{\circ} \mathrm{C}$ and $27^{\circ} \mathrm{C}$. When the temperature and humidity inside the chamber was at steady state, the experiments were started. The experiment has been conducted on the domestic refrigerator at no load and load conditions.

In this paper we have simulated a simple refrigeration system as a model of an actual system. We have used MATLAB-Simulink with Thermo system Toolbox, originally developed at the University of Illinois at Urbana-Champaign as a tool for simulating the transient performance of sub-critical and trans-critical vapor compression systems. Proposed model has been shown in figure. We have considered five inputs to system that are listed in below: 1.compressor inlet temperature,2.condenser out let temperature, 3.Condenser air mass flow rate, 4.evaporator air mass flow rate,5.evaporator air inlet temperature. Important parameters that are considered as outputs in proposed system arecop, minimize the energy consumption, condenser pressure, evaporator pressure, and evaporator air outlet temperature.

As we stated above, the inputs of our network are refrigerant mixtures of varying ratios and the outputs are the coefficient of performance and the minimization the input energy of the refrigeration system.

As mentioned earlier, we have considered using HFC and $\mathrm{HC}$ based refrigerant mixtures instead of CFCs (R12, R22, and R502). In previous studies, the following mixtures were suggested as substitutes for R12, R22, and 
R502. They can be listed as: Substitutes for R134a: R290/R600a (40/60, 43/57, 48/52, 50/50, 56/44, 60/40, 70/30, 80/20, 90/10),

For ANNs, two data-sets are needed: one for training the network and the second for testing it. The usual approach is to prepare a single data-set. From this section the comparison of the performance parameter of the refrigerants and energy consumption by the refrigerator was discussed this investigation deals with mixed refrigerant (hydrocarbon mixtures of propane, butane and isobutene) in order to assess their feasibility for replacing HFC-134a in refrigeration systems by comparing their relevant parameters.

The refrigerating effect is the main purposes of the refrigeration system. The liquid refrigerant at low pressure side enters the evaporator. As the liquid refrigerant passes through the evaporator coil, it continually absorbs heat through the coil walls, from the medium being cooled. During this, the refrigerant continues to boil and evaporate. Finally the entire refrigerants have evaporated and only vapor refrigerant remains in the evaporator coil.

The liquid refrigerant still colder than the medium being cooled, therefore the vapor refrigerants continue to absorb heat. The experiment was performed on the domestic refrigerator purchased from the market, the components of the refrigerator was not changed or modified. This indicates the possibility of using mixed refrigerant as an alternative of HFC-134a in the existing refrigerator system. Freezer temperature was measured at the different time interval and also observed the lowest temperature level.

\subsection{Selection of Patterns for Training}

The numbers of classes (Range of COPs), which are based on the classification range of the outputs, are decided. If only one output is considered the range of classification is simple. If more than one output is considered a combination criterion has to be considered. The total number of patterns is decided for each class. Out of these patterns, the number of patterns to be used for training the network is decided. The remaining patterns are used for testing the classification performance of the network. The patterns selected for training the network should be, such that they represent the entire population of the data.

\subsection{Back Propagation Algorithm}

The BPA uses the steepest-descent method to reach a global minimum. Flow-chart of the BPA is given in Figure 2 . The number of layers and number of nodes in the hidden layers are decided. The connections between nodes are initialized with random weights. A pattern from the training set is presented in the input layer of the network and the error at the output layer is calculated. The error is propagated backwards towards the input layer and the weights are updated. This procedure is repeated for all the training patterns. At the end of each iteration, test patterns are presented to ANN and the classification performance of ANN is evaluated. Further training of ANN is continued till the desired classification performance is reached. And also used fuzzy logic theory, finding the above output parameters.

\section{Result and discussion}

In this study, optimization of single-stage vapor compression refrigeration system using NL was carried out with R-134a and mixed refrigerants are used. In addition, thermodynamic properties of these refrigerants were obtained using FL. Enthalpies of different points of refrigerants are used in vapor compression refrigeration systems are predicted using FL approach. The temperature and pressure are the input data and enthalpy of the refrigerants is the actual output. Models are run in MATLAB Fuzzy Logic Toolbox. In this study, Mamdani type fuzzy inference system (FIS) was employed. And also study of ANN with BPA / RBF for finding out optimum mixed refrigerants to achieve very high $\mathrm{COP}$ and minimized the energy consumption. Data have been simulated to train and test the performance of ANN algorithms and fuzzy logic theory. The BPA requires some iteration for however; $\mathrm{RBF}$ requires one iteration to learn all the training patterns. This is a major advantage of RBF over BPA.RBF can produce a better result when compared to BPA to estimation of COP. The number of computational complexity is more for BPA than that of RBF.

\section{Conclusion}

This paper invested an ozone friendly, energy efficient, user friendly, safe and cost-effective alternative refrigerant for HFC134a using domestic refrigeration systems.

1) After the successful investigation on the performance of mixed refrigerants the following conclusions can be drawn based on the results obtained.

2) This experimental investigation carried out to determine the performance of a domestic refrigerator when a propane/butane mixture is used as a possible replacement to the traditional refrigerant for R134a. In this research various pressure values are observed and compared with the sole refrigerant (R134a) and mixed refrigerant (Propane - Butane Mixture).

3) The subsequent conclusions can be elicited from our research, i.e., Each and every loading conditions of mixed refrigerant (Propane-Butane) yields higher performance of cooling effect while compared with R134a.

4) Using the mixed refrigerant in domestic refrigerator, we were observed the freezer temperature is lower than that of the R134a. A smart observation was found in the loading of vegetables at the up and down the cooling effect.

\section{Nomenclature}

ANN Artificial Neural Network

BPA Back-Propagation algorithm

FL Fuzzy Logic

FPGA Field Programmable Gate Array

FSO Full-Scale Output 


\author{
LMS Least Mean Square \\ MLP Multi-Layer Perception \\ MR Measured Range \\ MSE Mean Square Error \\ LPH Liter per Hour \\ PIM Plug-In-Module \\ RBFNN Radial Basis Function based Neural Network \\ ANN Artificial Neural-Network \\ CFC Chlorofluorocarbon \\ COP Coefficient of Performance \\ $\mathrm{HC}$ Hydrocarbon \\ HFC Hydro fluorocarbon \\ Q Heat load \\ $\mathrm{R}^{2} \quad$ Fraction of variance \\ RMS Root-Mean-Square error \\ $\mathrm{T} \quad$ Temperature, ${ }^{\circ} \mathrm{C}$ \\ TI Total irreversibility $(\mathrm{kJ} / \mathrm{kg})$

\section{Subscripts and superscripts:} \\ a Air \\ c Condenser \\ e Evaporator \\ CFCS Chlorofluorocarbons \\ GWP Global warming potential \\ HCFCs Hydro chlorofluorocarbons \\ HCs Hydrocarbons \\ HFCs Hydro fluorocarbons \\ ODP Ozone depletion potential \\ $\mathrm{P} \quad$ Pressure $\mathrm{kPa}$ \\ RE Refrigerating effect, $\mathrm{kJ} \mathrm{Kg-1}$ \\ MFR Mass flow rate, kgs-1
}

\section{References}

Swati Jain (2013)Soft Computing, Artificial Intelligence, Fuzzy Logic \& Genetic Algorithm in Bioinformatics Abhishek Pandey Faculty of CS, Takshshila Institute of Engineering \& Technology, Jabalpur. IJCEM International Journal of Computational Engineering \& Management, Vol. 16 Issue 1
G.Kumaresan (2013)Optimizing Design Of Heat Pump Using Fuzzy Logic And Genetic Algorithm International Journal of Engineering Research and Applications (IJERA) ISSN: 22489622 Vol. 3, Issue 3, May-Jun 2013, pp.1184-1189

Shivani (2012) Application of Soft Computing Techniques: Fuzzy logic and Genetic Algorithms. Principles of Soft Computing. IJSRET volume 1 issue 5 pp 309-312.

A.Akash, S.A. Said, (2003). Assessment of LPG as a possible alternative to R-12 in domestic refrigerators. Energy conversion and Management, Vol. 44, pp. 381-388

Chen, S., and Billings, S.A, (1992), Neural networks for nonlinear dynamic system modeling and identification, International Journal of Control, Vol.56, No. 22, pp. 319-349

Gunther D, Steimle F., (1997), Mixing rules for the specific heat capacities of several HFC-mixtures. International Journal of Refrigeration, Vol. 20, pp. 235-43

Joseph Sekhar, D.MohanLal, S. Renganarayanan., (2004), Improved energy efficiency for $\mathrm{CFC}$ domestic refrigerators with ozone-friendly $\mathrm{HFC} 134 \mathrm{a} / \mathrm{HC}$ refrigerant mixture, International Journal of thermal Science, Vol. 43, pp. 307-31

Jung D, Kim CB, Song K, Park B., (2000), Testing of propane/isobutene mixture in domestic refrigerators. Int. $J$ Refrig. Vol. 23, No. 7, pp. 517-527

N.Austin,P.Senthilkumar,N.Kanthavelkumaran., (2012), Study of Thermodynamic Optimization Criteria for Domestic Refrigerator (propane -butane as diverse refrigerant), International Journal of Mechanical and Production Engineering Research and Development (IJMPERD), ISSN 2249-6890, Vol. 2 Issue 4, pp. 83-8

R. Radermacher, K. Kim., (1996), Domestic refrigerator: recent development, International journal of refrigeration Vol, 19, 61-6

Sharma, R., Singhal, D., Ghosh, R., and Dwivedi., A., (1999). Potential applications of artificial neural networks to thermodynamics: vapor-liquid equilibrium predictions. Computers and Chemical Engineering. Vol. 23, pp. 385-390

M.Mohanraj,S.Jaraj,C.Muraleedharan(2012),Applications of artificial neural networks for refrigeration, air-conditioning and heat pump systems-A review Elsevier renewable and sustainable energy reviews 16 pp 1340-1358 